\title{
Experiments on Rest-to-rest Motion of a Flexible Arm
}

\author{
Alessandro De Luca ${ }^{1}$, Valerio Caiano ${ }^{2}$, and Dionisio Del Vescovo ${ }^{2}$ \\ 1 Dipartimento di Informatica e Sistemistica (DIS) and \\ 2 Dipartimento di Meccanica e Aeronautica (DMA) \\ Università di Roma "La Sapienza" \\ Via Eudossiana 18, 00184 Roma, Italy \\ deluca@dis.uniroma1.it
}

\begin{abstract}
We present an experimental validation of a recently proposed solution to the problem of finding the input torque command that provides rest-to-rest motion in a given time for a one-link flexible arm. The basic idea is to design an auxiliary output such that the associated input-output transfer function has no zeros. Planning a smooth interpolating trajectory for this output imposes a unique rest-to-rest motion to the whole arm, with automatically bounded link deformations. The nominal torque is then obtained by standard inverse dynamics computation. The method is presented for a linear model of an Euler-Bernoulli flexible beam, satisfying dynamic boundary conditions and taking into account also modal damping. We illustrate the dynamic identification of the experimental flexible arm, the handling of static/viscous joint friction within the proposed method, and the way to include a stabilizing feedback based only on joint measurements. Finally, we report on comparative experimental results.
\end{abstract}

\section{Introduction}

Consider the basic problem of moving a flexible manipulator from one equilibrium configuration to another in a prescribed time. This problem arises when fast and precise end-effector positioning of a long reach lightweight arm is desired [1]. The gross (rigid) motion typically induces residual oscillations at the nominal task completion time, delaying the final instant at which the arm can be considered at rest. Adding dissipative feedback control [2] or relying on intrisic structural damping [3] are only partial remedies, while the design of a suitable feedforward motion command has been recognized as a critical step.

Two main model-based approaches have been proposed at the beginning of the 90's for designing a feedforward input solving the rest-to-rest motion problem in flexible mechanical systems (in particular, manipulators): input shaping [4,5] and inverse dynamics trajectory design [6,7]. Input shaping consists in convolving the reference command (typically, a step input) with impulses, suitably located in time, that 'kill' the modal frequencies of the flexible arm. The method is straightforward for one or few flexible modes [4], but more complex when increasing the number of considered modes or for achieving robustness [5]. It also introduces some delay in the actual system response. On the other hand, one can design a smooth interpolating trajectory for the end-effector of a flexible arm and then use stable (i.e., with bounded 
link deformations) input-output inversion for computing the rest-to-rest torque command in the frequency [6] or time domain [7]. However, the resulting torques are non-causal, extending in time both before the start and after the completion of the planned end-effector trajectory, while practical accuracy and exact motion timing are limited by the finite window in time (or in frequency) used in the implementation. Residual vibration suppression has been obtained with a different method in [8]. A combination of sinusoidal components is used to build up a rest-to-rest trajectory, from which the nominal torque is computed by inversion: the solution of a linear algebraic system is needed, which becomes ill-conditioned for increasing number of flexible modes.

In [9], a novel approach has been presented for a one-link flexible arm. The main idea was to design an auxiliary output with maximum relative degree, namely such that the input torque affects only the highest-order time derivative of the output. When facing a scalar flexible system with linear dynamics, this is equivalent to obtaining a transfer function between the input torque and the defined output that has no zeros. The rest-to-rest motion problem can be then easily solved by fitting to this output a smooth polynomial that interpolates the start and final rest configurations. In addition, the design output and the associated nominal rest-to-rest torque obtained by system inversion are both determined in closed form for any number of flexible modes.

In this paper, we show the practical feasibility and performance of the method proposed in [9], by addressing the following additional issues for a one-link flexible arm:

- Inclusion of modal damping in the method

- Handling of static/viscous friction at the joint

- Identification of the dynamic model

- Addition of a robustifying feedback based only on joint measurements

- Experimental validation and performance comparison

The paper is organized as follows. In Sect. 2 we shortly review the dynamic modeling. The design method in the frequency domain is described in Sect. 3, including the presence of modal damping. Section 4 presents the dynamic identification of our flexible arm and some comparative experimental results on a rather fast rest-to-rest motion.

\section{Dynamic Modeling}

We consider a one-link flexible arm, of length $\ell$ and uniform linear mass density $\rho$, rotating on a horizontal plane. The arm is driven by an actuator at the base, with inertia $J_{0}$ and torque $\tau(t)$, and may carry a tip payload of mass $M_{p}$ and inertia $J_{p}$. The flexible link is modeled as an Euler-Bernoulli beam with Young modulus $E$ and inertia of the cross section $I$, assuming small deformations limited to the plane of motion. Let $\theta(t)$ be the angle to the instantaneous center of mass of the link (see 
Fig. 1). The transversal bending deformation at a point $x \in[0, \ell]$ along the link is described by $w(x, t)$.

From Hamilton principle, the motion equations of the flexible arm are [10]

$$
E I w^{\prime \prime \prime \prime}(x, t)+\rho(\ddot{w}(x, t)+x \ddot{\theta}(t))=0, \quad \tau(t)-J \ddot{\theta}(t)=0,
$$

where $J=J_{0}+\left(\rho \ell^{3}\right) / 3+J_{p}+M_{p} \ell^{2}$ is the total inertia of the arm w.r.t. the joint axis, with associated dynamic boundary conditions given by

$$
\begin{aligned}
& w(0, t)=0 \quad E I w^{\prime \prime}(0, t)=J_{0}\left(\ddot{\theta}(t)+\ddot{w}^{\prime}(0, t)\right)-\tau(t) \\
& E I w^{\prime \prime}(\ell, t)=-J_{p}\left(\ddot{\theta}(t)+\ddot{w}^{\prime}(\ell, t)\right) \\
& E I w^{\prime \prime \prime}(\ell, t)=M_{p}(\ell \ddot{\theta}(t)+\ddot{w}(\ell, t)),
\end{aligned}
$$

in which a prime denotes the spatial derivative w.r.t. $x$. By separation in space and time, assuming a finite number $n_{e}$ of deformation mode shapes $\phi_{i}(x)$ with associated deformation coordinates $\delta_{i}(t)$,

$$
w(x, t)=\sum_{i=1}^{n_{e}} \phi_{i}(x) \delta_{i}(t)
$$

and imposing the boundary conditions (1), the mode shapes take the form

$$
\phi_{i}(x)=A_{i} \sin \left(\beta_{i} x\right)+B_{i} \cos \left(\beta_{i} x\right)+C_{i} \sinh \left(\beta_{i} x\right)+D_{i} \cosh \left(\beta_{i} x\right),
$$

where $\beta_{i}^{4}=\rho \omega_{i}^{2} / E I$ and $\beta_{1}, \ldots, \beta_{n_{e}}$ are the first $n_{e}$ roots of the following characteristic equation

$$
\begin{aligned}
& (c s h-s c h)-\frac{2 M_{p}}{\rho} \beta_{i} s s h-\frac{M_{p}}{\rho^{2}} \beta_{i}^{4}\left(J_{0}+J_{p}\right)(c s h-s c h)-\frac{2 J_{p}}{\rho} \beta_{i}^{3} c c h \\
& -\frac{J_{0}}{\rho} \beta_{i}^{3}(1+c c h)+\frac{J_{0} J_{p}}{\rho^{2}} \beta_{i}^{6}(c s h+s c h)-\frac{J_{0} J_{p} M_{p}}{\rho^{3}} \beta_{i}^{7}(1-c c h)=0,
\end{aligned}
$$

with $s=\sin \left(\beta_{i} \ell\right), c=\cos \left(\beta_{i} \ell\right), s h=\sinh \left(\beta_{i} \ell\right)$, and $c h=\cosh \left(\beta_{i} \ell\right)$. The coefficients $A_{i}, \ldots, D_{i}$ are determined up to a normalization factor and the $\omega_{i}$ are the eigenfrequencies of the flexible arm, for $i=1, \ldots, n_{e}$.

The resulting Euler-Lagrange equations for the $N=n_{e}+1$ generalized coordinates $q=(\theta, \delta)=\left(\theta, \delta_{1}, \ldots, \delta_{n_{e}}\right)$ are

$$
\begin{aligned}
J \ddot{\theta} & =\tau \\
\ddot{\delta}_{i}+\omega_{i}^{2} \delta_{i} & =\phi_{i}^{\prime}(0) \tau, \quad 1, \ldots, n_{e} .
\end{aligned}
$$

Modal damping is taken into account by modifying eqs. (4) as

$$
\ddot{\delta}_{i}+2 \zeta_{i} \omega_{i} \dot{\delta}_{i}+\omega_{i}^{2} \delta_{i}=\phi_{i}^{\prime}(0) \tau, \quad 1, \ldots, n_{e},
$$

where $\zeta_{i} \in[0,1)$ are the non-dimensional damping coefficients.

Note finally that the two transfer functions $\tau \rightarrow \theta_{c}$ (clamped joint angle output) and $\tau \rightarrow \theta_{t}$ (tip angle output), where (see Fig. 1)

$$
\theta_{c}=\theta+\sum_{i=1}^{n_{e}} \phi_{i}^{\prime}(0) \delta_{i}, \quad \theta_{t}=\theta+\sum_{i=1}^{n_{e}} \frac{\phi_{i}(\ell)}{\ell} \delta_{i}
$$


are, respectively, minimum phase (strictly, if $\zeta_{i}>0$ ), i.e., with zeros only in the (open) left-hand side of the complex plane, and non-minimum phase ${ }^{1}$.

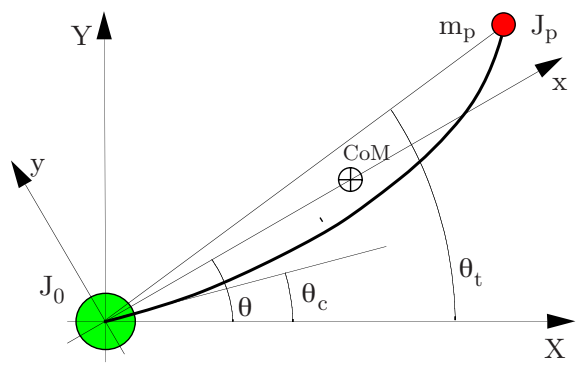

Fig. 1. Definition of variables for a one-link flexible arm

\section{Design Method}

Consider a rest-to-rest motion task for the one-link flexible arm modeled by eqs. (3) and (5). The arm should be moved from an initial undeformed configuration $q_{i}=$ $\left(\theta_{i}, 0\right)$ at time $t_{i}=0$ to a final undeformed configuration $q_{f}=\left(\theta_{f}, 0\right)$ at time $t_{f}=T$, with $\dot{q}(0)=\dot{q}(T)=0$.

We solve this planning problem by designing a suitable output function $y$ such that the associated transfer function $\tau \rightarrow y$ will have no zeros. This design output should have the form

$$
y=\theta+\sum_{i=1}^{n_{e}} c_{i} \delta_{i}+\gamma \dot{\theta}+\sum_{i=1}^{n_{e}} d_{i} \dot{\delta}_{i}
$$

with the coefficients $\gamma, c_{i}$, and $d_{i}\left(i=1, \ldots, n_{e}\right)$ to be determined by imposing the condition that the output (7) has maximum relative degree (equal to the state space dimension $2\left(n_{e}+1\right)$ of the flexible arm), i.e., $y$ and its first $2 n_{e}+1$ derivatives are independent from the input $\tau$. The actual computation of this output can be performed in the time domain (in fact, this may allow the generalization to a nonlinear setting). Details for the case of no modal damping are given in [9]. However, one can exploit the linearity of the system dynamics and work in the Laplace domain. We present here the extension of this closed-form calculation when including modal damping, namely for eqs. (3) and (5).

\footnotetext{
${ }^{1}$ From the boundary conditions (1), one has $\phi_{i}^{\prime}(0)=\lim _{x \rightarrow 0} \frac{\phi_{i}(x)}{x}$.
} 
The transfer function associated to the input-output map $\tau \rightarrow y$ is derived from eqs. (3), (5), and (7), and then equated to one having no zeros, i.e.,

$$
\frac{y(s)}{\tau(s)}=\frac{\gamma s+1}{J s^{2}}+\sum_{i=1}^{n_{e}} \frac{\left(d_{i} s+c_{i}\right) \phi_{i}^{\prime}(0)}{s^{2}+2 \zeta_{i} \omega_{i} s+\omega_{i}^{2}}=\frac{K}{s^{2} \prod_{i=1}^{n_{e}}\left(s^{2}+2 \zeta_{i} \omega_{i} s+\omega_{i}^{2}\right)},
$$

for a suitable scalar $K$. The unknown coefficients in eq. (8) are determined by using partial fraction expansions of the last two terms and imposing their equality. We obtain the following expressions:

$$
\begin{aligned}
K & =\frac{1}{J} \prod_{i=1}^{n_{e}} \omega_{i}^{2} & c_{i} & =\frac{2 \omega_{i}}{\phi_{i}^{\prime}(0)}\left[a_{i} \zeta_{i}-b_{i} \sqrt{1-\zeta_{i}^{2}}\right], \quad i=1, \ldots, n_{e} \\
\gamma & =-\sum_{i=1}^{n_{e}} \frac{2 \zeta_{i}}{\omega_{i}} & d_{i} & =\frac{2 a_{i}}{\phi_{i}^{\prime}(0)}, \quad i=1, \ldots, n_{e},
\end{aligned}
$$

where $R_{i}=a_{i} \pm j b_{i}$ are the (complex conjugate) residuals associated to the poles $\left(-\zeta_{i} \pm j \sqrt{1-\zeta_{i}^{2}}\right) \omega_{i}$, for $i=1, \ldots, n_{e}$. The real and imaginary parts of $R_{i}$ are computed as follows. For $i, k=1, \ldots, n_{e}$ (with $k \neq i$ ), let

$$
\begin{aligned}
\rho_{i} & =4 \omega_{i}^{3} \zeta_{i}\left(1-\zeta_{i}^{2}\right) & v_{i k} & =\omega_{i}^{2}\left(2 \zeta_{i}^{2}-1\right)+\omega_{k}^{2}-2 \omega_{i} \omega_{k} \zeta_{i} \zeta_{k} \\
\sigma_{i} & =2 \omega_{i}^{3}\left(2 \zeta_{i}^{2}-1\right) \sqrt{1-\zeta_{i}^{2}} & w_{i k} & =2 \omega_{i} \sqrt{1-\zeta_{i}^{2}}\left(\omega_{k} \zeta_{k}-\omega_{i} \zeta_{i}\right)
\end{aligned}
$$

and

$$
\Delta_{i}=\frac{K}{\left(\rho_{i}^{2}+\sigma_{i}^{2}\right) \prod_{k \neq i}^{n_{e}}\left(v_{i k}^{2}+w_{i k}^{2}\right)} \quad \Gamma_{i}=\left(\rho_{i}-j \sigma_{i}\right) \prod_{k \neq i}^{n_{e}}\left(v_{i k}-j w_{i k}\right) .
$$

We finally have, for $i=1, \ldots, n_{e}, a_{i}=\Delta_{i} \cdot \operatorname{Re}\left[\Gamma_{i}\right]$ and $b_{i}=\Delta_{i} \cdot \operatorname{Im}\left[\Gamma_{i}\right]$. The previous formulas determine uniquely the design output. In the absence of modal damping $\left(\zeta_{i}=0\right.$, for all $\left.i\right)$, it is easy to verify that the output coefficients reduce to the expressions given in [9]:

$$
\gamma=0, \quad d_{i}=0, \quad c_{i}=-\frac{1}{J \phi_{i}^{\prime}(0)} \prod_{\substack{j=1 \\ j \neq i}}^{n_{e}} \frac{\omega_{j}^{2}}{\omega_{j}^{2}-\omega_{i}^{2}}, \quad i=1, \ldots, n_{e} .
$$

Once the output has been defined according to eqs. (9), one can establish a transformation between the state $(q, \dot{q})=(\theta, \delta, \dot{\theta}, \dot{\delta})$ and the output $y$ with their time derivatives up to the $\left(2 n_{e}+1\right)$-th order, as

$$
\left[\begin{array}{llll}
y & \dot{y} & \ldots & y^{\left[2 n_{e}+1\right]}
\end{array}\right]=\left[\begin{array}{llll}
\theta & \delta^{T} & \dot{\theta} & \dot{\delta}^{T}
\end{array}\right] Q,
$$

with the invertible matrix $Q$ depending on the coefficients $c_{i}$ 's and $d_{i}$ 's. In particular, it can be shown that a generic undeformed configuration at rest $(\delta=0$, and $\dot{\theta}=\dot{\delta}=$ $0)$ maps into $y=\theta$ and $\dot{y}=\ddot{y}=\ldots=y^{\left[2 n_{e}+1\right]}=0$.

The rest-to-rest motion problem can then be solved by defining an interpolating trajectory $y=y_{d}(t), t \in\left[t_{i}, t_{f}\right]$, with appropriate boundary conditions at time $t_{i}=0$ and $t_{f}=T$. From the structure of eq. (10), it is enough to set $y_{d}(0)=\theta_{i}$, 
$y_{d}(T)=\theta_{f}$, with all derivatives up to the $\left(2 n_{e}+1\right)$-th order equal to zero at the initial and final time. For satisfying these boundary conditions, a polynomial of degree $4 n_{e}+3$ will be sufficient. Choosing polynomials of higher (odd) degree, with symmetric zero boundary conditions on the derivatives at initial and final times, provides further desired smoothness of the nominal torque.

A closed-form expression of the nominal torque $\tau_{d}(t)$ can be obtained without the need of computing the coefficients $\gamma, c_{i}$ 's, and $d_{i}$ 's. In fact, setting $y=y_{d}$ in eq. (8) and using $K$ from eqs. (9), we obtain in the Laplace domain

$$
\tau_{d}(s)=\frac{J}{\prod_{i=1}^{n_{e}} \omega_{i}^{2}}\left[s^{2} \prod_{i=1}^{n_{e}}\left(s^{2}+2 \zeta_{i} \omega_{i} s+\omega_{i}^{2}\right)\right] y_{d}(s)
$$

and thus in the time domain

$$
\tau_{d}(t)=\frac{J}{\prod_{i=1}^{n_{e}} \omega_{i}^{2}}\left[y_{d}^{\left[2\left(n_{e}+1\right)\right]}(t)+\sum_{i=0}^{2 n_{e}-1} \alpha_{i} y_{d}^{[i+2]}(t)\right],
$$

with real constants $\alpha_{i}$ easily obtained by convolution of polynomial coefficients. In the absence of modal damping only even derivatives of the output trajectory appear.

It is interesting to note that the designed output has a clear physical interpretation in case of no modal damping $\left(\gamma=0, d_{i}=0, i=1, \ldots, n_{e}\right)$. In analogy with the clamped joint and tip angle outputs (6), the output function (7) is the angle to a specific point along the link located at a distance $x=x^{*} \leq \ell$ from the base:

$$
y=\theta+\sum_{i=1}^{n_{e}} c_{i} \delta_{i}=\theta+\sum_{i=1}^{n_{e}} \frac{\phi_{i}\left(x^{*}\right)}{x^{*}} \delta_{i} .
$$

At $x^{*}$, there is a transition between stable and unstable inverse dynamics. In fact, while displacing $x$ from 0 to $\ell$, the zeros of the input-output transfer function slide along the imaginary axis (passive minimum-phase output $\theta_{c}$ ), overpassing the location of the poles (losing the passivity property) and then going to infinity (vanishing) for $x=x^{*}$ (design output $y$ ). Beyond this point, the zeros of the transfer function reappear in symmetric positive/negative real pairs (non-minimum phase output $\theta_{t}$ ).

Finally, in a real implementation it is convenient to add a feedback action that stabilizes the flexible arm along the nominal trajectory. This can be achieved by a purely joint-based PD controller (see, e.g., [11])

$$
\tau=\tau_{d}+k_{p}\left(\theta_{c, d}-\theta_{c}\right)+k_{d}\left(\dot{\theta}_{c, d}-\dot{\theta}_{c}\right),
$$

where $k_{p}>0, k_{d}>0, \tau_{d}(t)$ is given by eq. (11), and $\theta_{c, d}(t)$ is the reference trajectory for the clamped joint angle (directly measured by the motor encoder). This joint reference trajectory is computed off-line either in an algebraic way, by inverting eq. (10) for $y=y_{d}(t)$ and using eq. (6), or by simulation of the system dynamics (3) and (5) under the nominal torque $\tau_{d}(t)$. 


\section{Experimental Results}

We have implemented the rest-to-rest method on the flexible arm available at the DMA of our University (see Fig. 2). The arm has length $\ell=0.655 \mathrm{~m}$ and is made of an harmonic steel sheet (2 mm thick and $51 \mathrm{~mm}$ wide) with measured $E I=6.22 \mathrm{Nm}^{2}$ and $\rho=0.7733 \mathrm{~kg} / \mathrm{m}$. The actuator is a current-driven DC motor directly coupled to the link with maximum torque of about $1 \mathrm{Nm}$. The motor inertia is $J_{0}=1.888 \cdot 10^{-3} \mathrm{~kg} \mathrm{~m}^{2}$, while its actual current-to-torque gain is $0.2966 \mathrm{Nm} / \mathrm{A}$. The arm has no tip payload $\left(M_{p}=J_{p}=0\right)$. The encoder mounted on the motor has a resolution of 4000 pulses/turn, amplified by a factor of 4 through electronics. Seven full bridges of strain gauges are mounted on the link in order to measure deformation. In this work, these measures have been used only for assessing the vibrations along the arm (and not for feedback). The used sampling frequency of the controller is $4 \mathrm{KHz}$.

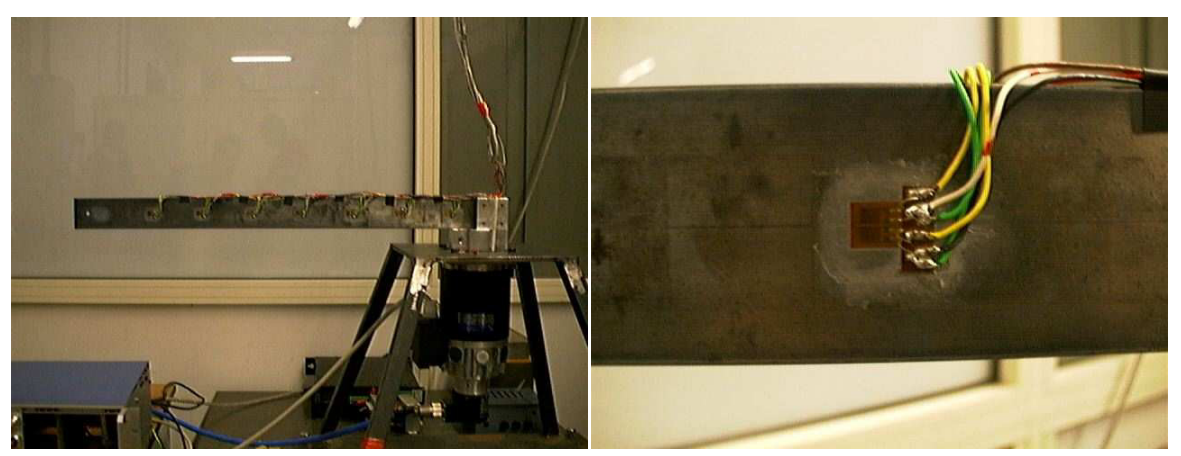

Fig. 2. The experimental one-link flexible arm and one of the seven strain gauges

Before applying the method, the friction acting at the joint needs to be taken into account. We have modeled it as

$$
\tau_{F}=F_{s} \operatorname{sign}\left(\dot{\theta}_{c}\right)+F_{v} \dot{\theta}_{c}
$$

where $F_{s}$ and $F_{v}$ are, respectively, the static and viscous friction coefficients. Friction has been identified separately, recording the current needed to keep the arm rotating at different constant speeds in a series of experiments. By linear regression we found $F_{s}=0.0504 \mathrm{Nm}$ and $F_{v}=0.0282 \mathrm{Nm} /(\mathrm{rad} / \mathrm{s})$. Since joint friction acts on the system in the same way as the input torque command, we can obtain (partial) friction compensation by adding to the nominal feedforward torque $\tau_{d}(t)$ the term

$$
\tau_{F, d}=F_{s} \operatorname{sign}\left(\dot{\theta}_{c, d}\right)+F_{v} \dot{\theta}_{c, d}
$$

In order to identify the dynamic model (3) and (5), we have excited the structure with a frequency sweep signal, with constant amplitude and frequency linearly increasing with time. Up to five resonant frequencies were identified using the 
MATLAB Frequency Domain System Identification toolbox. The first three modal eigenfrequencies and associated damping coefficients were found to be:

$$
f_{1}=14.4, f_{2}=34.2, f_{3}=69.3(H z) \quad \zeta_{1}=0.0001, \zeta_{2}=0.001, \zeta_{3}=0.008 .
$$

Using the experimental values $\omega_{i}=2 \pi f_{i}$ and the mode shape expressions (2), the associated mode shape spatial derivatives at the link base are:

$$
\phi_{1}^{\prime}(0)=11.7699, \quad \phi_{2}^{\prime}(0)=16.4439, \quad \phi_{3}^{\prime}(0)=8.6898 .
$$

Figure 3 shows the comparison of experimental vs. analytical frequency responses, providing a very good validation of the model. The errors between model-based and measured eigenfrequencies are below $1 \%$.

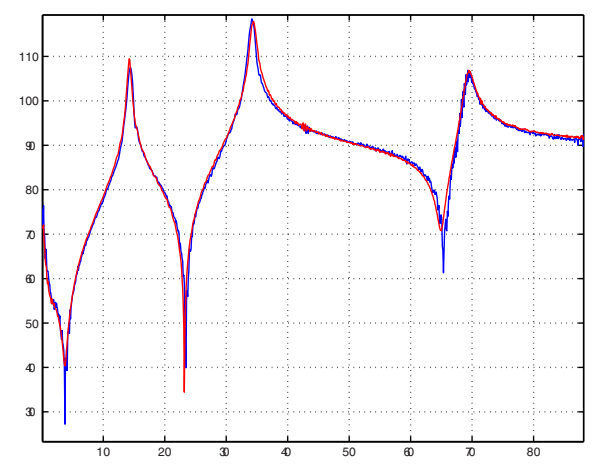

Fig. 3. Joint acceleration frequency response (plant vs. model) $[\mathrm{dB} / \mathrm{Hz}]$

We report here experimental results using only $n_{e}=3$ modes in the dynamic model and executing a slew of $180^{\circ}$ in $T=2.2 \mathrm{~s}$. The designed output trajectory is a 19th-degree polynomial, which guarantees also zero torque and zero time derivative of the torque at the initial and final instants.

The first set of plots show the typical performance of a pure feedforward strategy. The nominal torque in Fig. 4 is almost symmetric w.r.t. motion midtime, apart from the off-set due to the compensation of static friction (kept also beyond $T=2.2 \mathrm{~s}$ ). The measured rotation at the link base (the clamped joint angle in Fig. 5a) shows that a residual position error is left at the end of the motion. This is mainly due to the actual non-uniform friction along a full rotation of the arm. In other experiments, no residual error was found. Figure $5 b$ shows the bending angle at the tip w.r.t. the rigid angular motion (i.e., $\theta$ ).

The second set of results are obtained with the addition of a joint PD feedback as in eq. (12), with gains $k_{p}=36.25 \mathrm{Nm} / \mathrm{rad}$ and $k_{d}=0.02 \mathrm{Nm} /(\mathrm{rad} / \mathrm{s})$. The applied torque in Fig. 6a is more nervous but quite similar to the nominal one. The joint and tip evolutions (Fig. 7) are almost perfect, with a peak error of less than $0.15 \mathrm{deg}$ in both cases. In order to better evaluate the vibrational behavior of the arm, the bending 


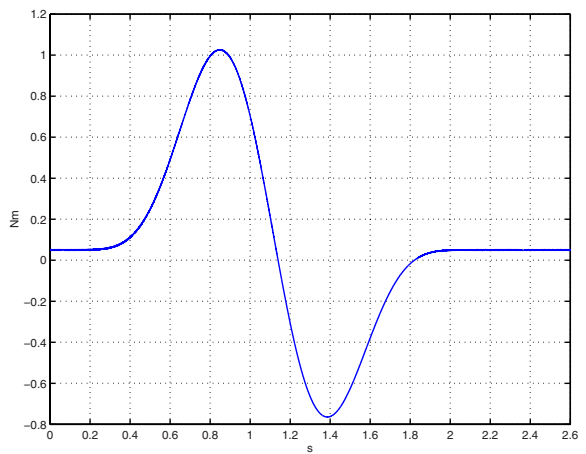

Fig. 4. Applied feedforward torque
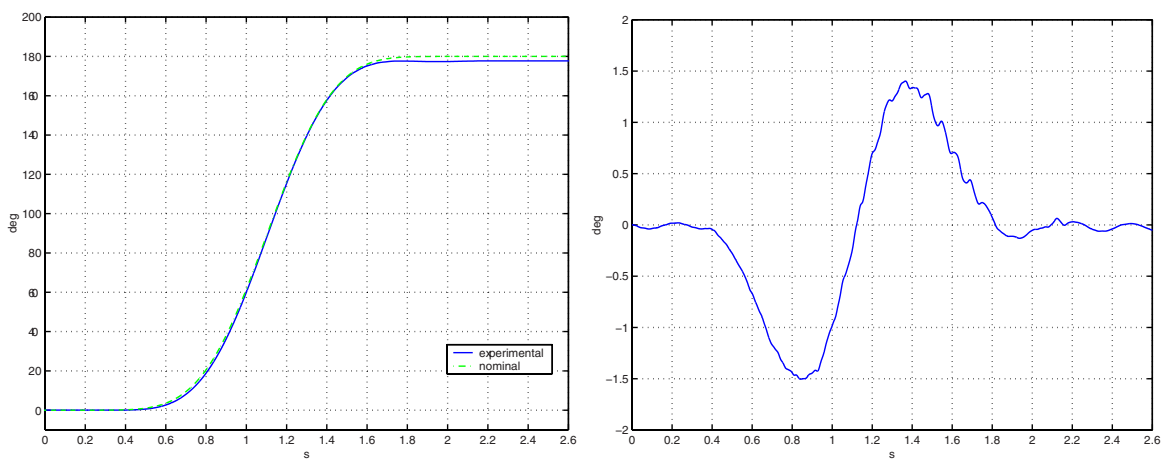

Fig. 5. Clamped joint angle (a). Bending deformation at the tip (b)
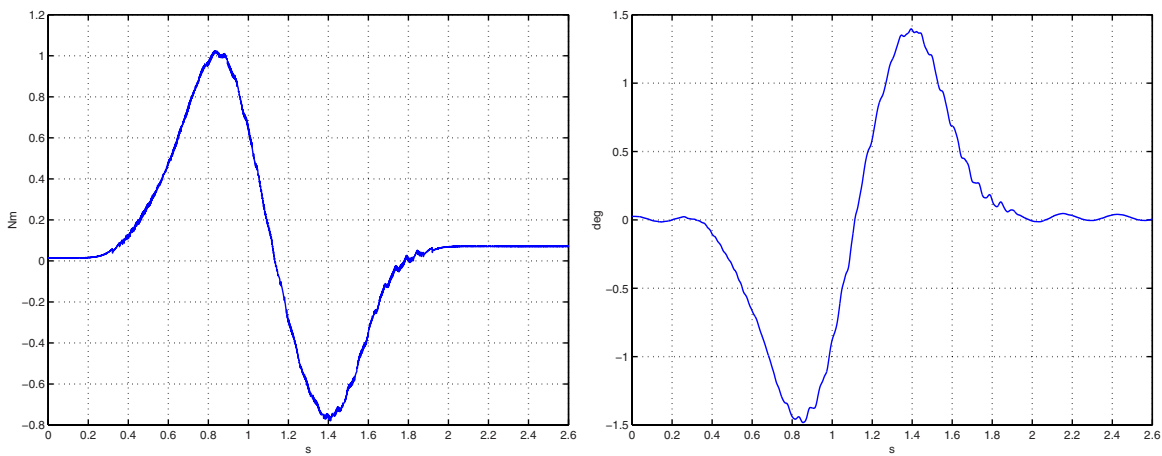

Fig. 6. Applied torque including joint PD feedback (a). Bending deformation at the tip (b) 

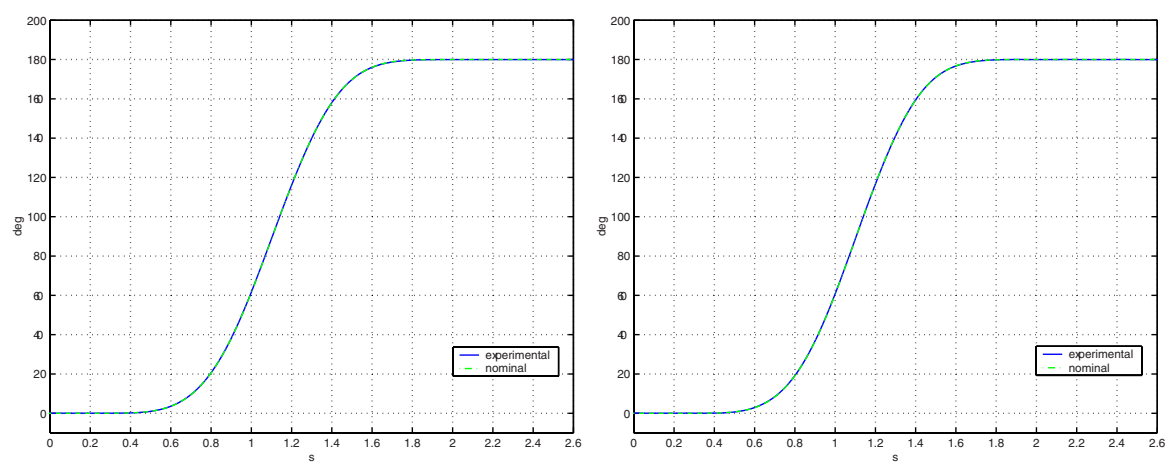

Fig. 7. Clamped joint angle (a). Tip angle (b)

deformation at the tip is also shown in Fig. 6b. These results are very repetitive in all performed experiments.

As a final comparative test, we have used the simple feedback controller

$$
\tau_{\text {comp }}=J \ddot{y}_{d}+k_{p}\left(y_{d}-\theta_{c}\right)+k_{d}\left(\dot{y}_{d}-\dot{\theta}_{c}\right)
$$

where $J, k_{p}$, and $k_{d}$ are the same as before, while $y_{d}(t)$ is a 7-th order polynomial with zero boundary conditions on velocity, acceleration, and jerk. We note that, apart from the simpler feedforward term (based here only on the rigid model), the main difference between eq. (12) and (14) is the use of the model-based computed reference $\theta_{c, d}(t)$ in place of $y_{d}(t)$. The nominal compensation of friction (13) is also added to $\tau_{\text {comp. }}$. Figure 8 shows that both the torque and the tip bending deformation are more affected from oscillations than before. Finally, Fig. 9 compares the tip acceleration profiles with the rest-to-rest method and when using the control law (14). In the presence of a maximum angular bending of $1.5 \mathrm{deg}$, the tip acceleration profile in Fig. 9a shows neglectable oscillations after $T=2.2 \mathrm{~s}$ with our method. On the other hand, although the applied torque and the tip bending deformation are both much smaller using (14), the tip angular acceleration in Fig. 9b is still considerably large after the nominal completion time, indicating the presence of undesired residual oscillations.

\section{Conclusions}

We have presented an experimental validation of a new method for computing the nominal torque that achieves rest-to-rest motion in given time for a one-link flexible arm, based on the definition of a design output with maximum relative degree. The obtained results show the feasibility of the method and its robustness, when adding a simple joint PD feedback, to unmodeled dynamics such as high-frequency modes and friction.

The same approach can be applied in principle to any controllable linear singleinput system. Interestingly enough, the time-based version of this design method 

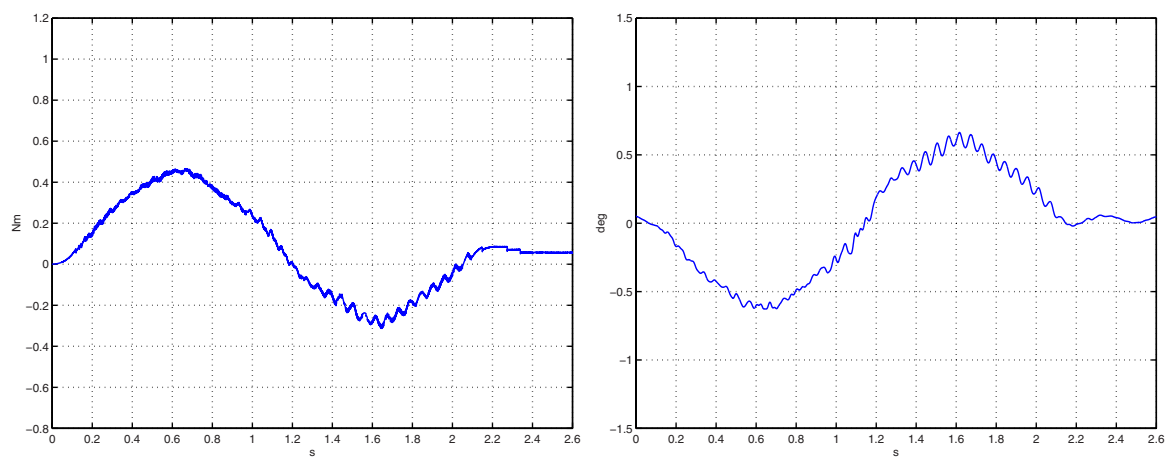

Fig. 8. Applied torque including PD feedback for a smooth rigid motion with 7th-order polynomial joint trajectory (a). Bending deformation at the tip (b)
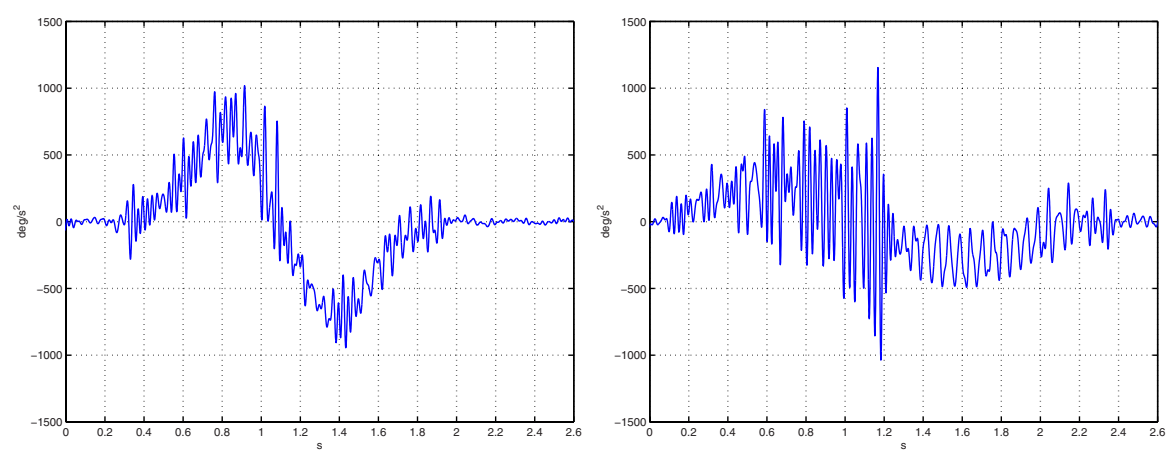

Fig. 9. Comparison of tip angle acceleration. Rest-to-rest method (a). Smooth rigid motion (b)

can be extended also to nonlinear multi-input systems, by finding an output vector without zero dynamics. A first example has been provided in [12], where rest-torest motions were successfully planned for a two-link planar robot with a flexible forearm.

In terms of residual vibrations, our method showed improvements in comparison with the use of smooth model-independent polynomials as reference trajectory for the flexible arm. The differences would become even larger if faster execution times were allowed by the actuator capabilities. Actually, the simple interpolation with high-order polynomials used within our method leads to large peak torque values attained only for few instants around the motion midpoint. However, the presented method can be modified so as to generate bang-bang or bang-coast-bang type torque profiles, with suitable interpolating phases near the start and final (and midway) instants. Preliminary results indicate that a reduction of about $70 \%$ (!) of the peak torque can be obtained in this way for the same motion time. 


\section{Acknowledgments.}

Work supported by MIUR within the MISTRAL project.

\section{References}

1. Book, W. J. (1990) Modeling, design, and control of flexible manipulator arms: A tutorial review. 29th IEEE Conf. on Decision and Control, 500-506

2. Cetinkunt, S., Yu, W.-L. (1991) Closed-loop behavior of a feedback-controller of a flexible arm: A comparative study. Int. J. of Robotics Research, 10(3), 263-275

3. Kung, S. W., Singh, R. (1998) Vibration analysis of beams with multiple constrained layer damping patches. J. of Sound and Vibration, 212(5), 781-805

4. Singer, N. C, Seering, W. P. (1990) Preshaping command inputs to reduce system vibration. ASME J. of Dynamic Systems, Measurements, and Control, 112, 76-82

5. Hyde, J. M., Seering, W. P. (1991) Using input command pre-shaping to suppress multiple mode vibration. 1991 IEEE Int. Conf. on Robotics and Automation, 2604-2609

6. Bayo, E. (1987) A finite-element approach to control the end-point motion of a single-link flexible robot. J. of Robotic Systems, 4(1), 63-75

7. Kwon, D.-S., Book, W. J. (1990) An inverse dynamic method yielding flexible manipulator state trajectories. 1990 American Control Conf., 186-193

8. Yang, H., Krishnan, H., Ang Jr., M. H. (1997) A simple rest-to-rest control command for a flexible link robot. 1997 IEEE Int. Conf. on Robotics and Automation, 3312-3317

9. De Luca, A., Di Giovanni, G. (2001) Rest-to-rest motion of a one-link flexible forearm. 2001 IEEE/ASME Int. Conf. on Advanced Intelligent Mechatronics, 923-928

10. Barbieri, E., Özgüner, Ü. (1988) Unconstrained and constrained mode expansions for a flexible slewing link. ASME J. of Dynamic Systems, Measurement, and Control, 110, 416-421

11. De Luca, A. (2000) Feedforward/feedback laws for the control of flexible robots. 2000 IEEE Int. Conf. on Robotics and Automation, 233-240

12. De Luca, A., Di Giovanni, G. (2001) Rest-to-rest motion of a two-link robot with a flexible forearm. 2001 IEEE/ASME Int. Conf. on Advanced Intelligent Mechatronics, 929-935 